\title{
Señales atmosféricas y del hielo marino asociadas a ENOS en el sector norte de la península antártica
}

\author{
Atmospheric and sea ice signals associated with ENSO \\ in the northern sector of the antarctic peninsula
}

Jorge F. Carrasco ${ }^{1}$

\begin{abstract}
Resumen
Las señales atmosféricas asociadas a El Niño son débiles en el sector norte de la Península Antártica. Así lo revelan los registros medios mensuales de la precipitación acumulada, la presión atmosférica y la temperatura mínima del aire; medidos por las estaciones antárticas chilenas O'Higgins, Prat y Frei. La señal más clara es en la precipitación que muestra una disminución durante los eventos de El Niño intensos, junto a un incremento de la presión superficial. Sinópticamente, los análisis compuestos de anomalías de la presión a nivel mar y $500 \mathrm{hPa}$ revelan un predominio de una alta (baja) presión centrada al oeste de la Península Antártica durante un episodio de El Niño (La Niña). También, se manifiesta un aumento de la superficie de la concentración del hielo en el sector norte del Mar de Bellingshausen asociada con El Niño y una disminución con La Niña. En general, las señales atmosféricas asociadas a El Niño encontradas en las estaciones antárticas chilenas son más claramente percibidas que las señales de La Niña.
\end{abstract}

\section{Palabras clave:}

El Niño Oscilación del Sur, Península Antártica, parámetros atmosféricos, corriente en chorro

\section{Abstract}

The atmospheric signals associated with El Niño are weak in the northern sector of the Antarctic Peninsula. This is revealed by the monthly average of the precipitation, the atmospheric pressure and the minimum air temperature registered by the Antarctic Chilean stations: O'Higgins, Prat and
Frei. The signal is clearer in the precipitation that shows a decrease during strong El Niño events, along with an increase in sea-level pressure. Also, an increase in sea-ice concentration occurs associated with El Niño in the northern Bellingshausen Sea and a decrease with La Niña. Synoptically, a high (low) pressure centered to the west of the Antarctic Peninsula predominates during an El Niño (La Niña) episode. In general, the atmospheric signals of El Niño registered by the Chilean stations are more clearly observed than the signals of La Niña.

\section{Key words:}

El Niño Southern Oscilation, Antarctic Peninsula, air minimum temperature, sea ice, atmospheric parameters, hielo marino

\section{INTRODUCCIÓN}

El fenómeno de El Niño Oscilación del Sur (ENOS), que incluye su contraparte La Niña, es la variabilidad climática interanual más significativa que tiene lugar en el planeta. Ocurre en la cuenca ecuatorial del Océano Pacifico con alteraciones oceánicas y atmosféricas, cuyos efectos se hacen sentir más allá de los trópicos, en diversos lugares alrededor del planeta. La Antártida no está ajena a la discusión de si los efectos de El Niño y La Niña se hacen sentir o no en este lejano continente y cómo. Durante los ochentas, van Loon \& Shea

1 Universidad de Magallanes, Punta Arenas-Chile.

$\checkmark$ jorge.carrasco@umag.cl 
(1987) encontraron una relación inversa entre las anomalías estaciónales de presión al nivel del mar en las latitudes polares y ENOS con desfase negativo de un año. Luego, Carleton (1988) encontró una relación entre la Oscilación del Sur (OS) y la concentración del hielo marino que rodea la Antártica, en particular en el sector del Mar de Weddell. Resultados que fueron confirmados más tarde por los estudios de Gloersen (1995) y Simmonds y Jacka (1995). Además, Yuan (2004) sugiere que los cambios en la celda de Hadley, en la corriente en chorro subtropical y en el tren de ondas de Rossby asociados con El Niño; serían el puente que vinculan la forzante tropical con la formación y mantenimiento del Dipolo Antártico (ADP). El ADP es una variabilidad climática de latitudes altas en el sistema interactivo de la atmósfera-océano-hielo caracterizado por una relación de fase opuesta entre el hielo marino y las anomalías de la temperatura superficial del mar en el Océano Pacífico Sur y el Océano Atlántico Sur, y que responde a la forzante del ENOS (Yuan \& Martinson 2001).

Por su parte, White y Peterson (1996) sugirieron que existe una relación entre la variabilidad de la corriente circumpolar antártica y ENOS; mientras que, resultados de Savage et al. (1988) mostraron que las anomalías frías de la temperatura en el Polo Sur e incremento de los vientos superficiales descendente ocurrian con un año de desfase con ENOS. También, Smith y Stearns $(1993 a, b)$ relacionaron las anomalías climáticas de la presión y temperatura superficial en la Antártida con el Î́ndice de la Oscilación del Sur (IOS). Ellos, concluyeron que el comportamiento climático del continente Antártico puede ser responsable de mantener la alta de bloqueo de Nueva Zelanda afectando el lado Australiano de la Oscilación del Sur (OS), o en otras palabras, una vinculación con ENOS. De hecho, Renwick (1998) encontró una mayor frecuencia de situaciones de bloqueos atmosféricos en el Océano Pacífico Sur con la fase cálida de ENOS. El estudio acerca del bloqueo ocurrido en junio de 1982 realizado por Mo et al. (1987), ya había revelado que la amplitud y duración del bloqueo se debió en parte, por el aporte de una masa fría desplazada desde el continente Antártico. La relación Antártida y ENOS se da a través de la alta de bloqueo de Nueva
Zelanda y su relación con los cambios en el sector occidental del Pacífico, es decir, el lado Australiano de la OS. Además, estudios del comportamiento de la precipitación en la Antártida muestran una relación entre la variabilidad interanual y ENOS (Cullather et al. 1996; Bromwich et al. 2000), principalmente en la costa del pacífico de la Antártida Occidental. Para mayor información, Turner (2004) presenta un comprensivo resumen de El Niño y la Antártida que se recomienda al lector.

El propósito de esta revisión es ver el comportamiento climático del sector norte de la Península Antártica (PA) durante los mayores eventos del ENOS ocurridos en el último cuarto del siglo 20 incluyendo los eventos 1982/83, $1987 / 88$, 1991-95/96 y 1997/98. Además se incluye el evento 2015/16 ocurrido en lo que va del siglo 21 .

\section{METODOLOGÍA}

Para el estudio se utilizan los datos de precipitación, temperatura y presión de las estaciones antárticas chilenas Eduardo Freí (62 $25^{\circ} \mathrm{S}, 58^{\circ} 53^{\circ} \mathrm{O}, 47 \mathrm{msnm}$.), Arturo Prat $\left(62^{\circ} 30^{\circ} \mathrm{S}, \quad 59^{\circ} 41^{\circ} \mathrm{O}, 5 \mathrm{msnm}\right)$ y Bernardo

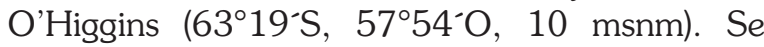
analizaron el comportamiento promedio mensual de la presión atmosférica a nivel medio del mar de la estación de Eduardo Freí (PresionFrei), las temperaturas mínimas promediadas de las estaciones Eduardo Freí, Arturo Prat y Bernardo O'Higgins (PromTmin); y el promedio de las precipitaciones mensuales acumuladas de estas tres estaciones antárticas (PromPrecip). Para ello, se obtuvieron las anomalías de la presión, temperatura y precipitación respecto al promedio del periodo 1970-2000. Solo la presión atmosférica medida en la estación Frei fue utilizada ya que esta variable puede representar el comportamiento local en el sector norte de la península. Por otro lado, se usa la temperatura mínima de las tres estaciones para representar su comportamiento medio en el sector. No se consideró usar la temperatura media ya que la temperatura máxima puede ser más variable entre las estaciones por diferencias de exposición a la radiación solar, no así la temperatura mínima. En todo caso, se estima que los resultados no difieren 
significativamente si se realizan con las temperaturas medias. Para analizar el comportamiento de la precipitación en el sector norte de la península se consideró mejor obtener el promedio mensual de la precipitación registrada en cada estación. El Índice de la Oscilación del Sur (IOS) es calculado por la diferencia de los valores mensuales de la presión a nivel del mar entre las mediciones de las estaciones Tahití y Darwin (Australia) (Chen, 1982, Trenberth, 1997). Aquí se evaluaron las anomalías estandarizadas. De este modo se obtuvieron los episodios indicados en la Tabla 1. Se considera la ocurrencia de un evento El Niño o La Niña si el IOS es, respectivamente, menor que -0.5 ó mayor que 0.5 . Tanto las anomalías de la presión, temperatura mínima, precipitación y del IOS fueron suavizadas utilizando un filtrado exponencial (ver anexo) con el fin de filtrar la alta variabilidad mensual.

Para un análisis de la circulación atmosférica se recurre a los Reanálisis de NCEP/NCAR (20th Century Reanálisis) disponible en la página web del Laboratorio de Investigación del Sistema Tierra (Earth System Research Laboratory) de la NOAA (www.esrl.noaa.gov) y la página Climate Reanalyzer (cci-reanalyzer.org) para la construcción de los campos de anomalías compuestas para los episodios El Niño y La Niña. Análisis de anomalías compuestas para los principales años de El Niño (1982, 1987, 1994 y 1997, 2015) y de La Niña (1988, 1996, 1998, 2008, 2010, 2011 y 2013) ocurridos entre los años 1970 y 2017 se obtuvieron para el período abril - agosto (otoñoinvierno) y septiembre - marzo (primavera-verano) de los campos de presión atmosférica a nivel medio del mar (mapa de superficie), altura geopotencial de $500 \mathrm{hPa}$ (nivel medio de la troposfera) y velocidad del viento zonal a $250 \mathrm{hPa}$ (troposfera alta). El episodio de El Niño 1991-1995 es representado sólo por el año 1994 en los análisis compuestos, para no sobre pesar el promedio en comparación a los años individuales de los otros episodios. Las anomalías compuestas construidos a partir de los datos de reanálisis son respecto a la media del periodo 1981-2010.

Los datos de concentración de hielo marino (CHM) fueron obtenidos de la página http://www. metoffice.gov.uk/ adobs/hadisst2/data/download. html, de la base de datos de observaciones de la Oficina de Meteorología del Centro Hadley (MetOffice Hadley observations datasets). Se obtuvo

Tabla 1. Años de eventos Niños y Niñas para períodos de invierno y verano. Cifras destacadas señalan intensidad moderada a fuerte

\begin{tabular}{|c|c|c|c|}
\hline Niño invierno & Niño verano & Niña invierno & Niña verano \\
\hline \multirow[t]{3}{*}{1972} & $1972 / 73$ & 1973 & $1973 / 74$ \\
\hline & & 1974 & \\
\hline & & 1975 & $1975 / 76$ \\
\hline \multirow[t]{2}{*}{1977} & $1977 / 78$ & & \\
\hline & & 1981 & \\
\hline \multirow[t]{2}{*}{1982} & $1982 / 83$ & & \\
\hline & $1986 / 87$ & & \\
\hline 1987 & & 1988 & 1988/89 \\
\hline 1991 & $1991 / 92$ & & \\
\hline 1993 & $1992 / 93$ & & \\
\hline 1994 & $1994 / 95$ & 1996 & \\
\hline \multirow[t]{4}{*}{1997} & $1997 / 98$ & 1998 & \\
\hline & & & 1998/99 \\
\hline & & & $1999 / 00$ \\
\hline & & & $2000 / 01$ \\
\hline \multirow[t]{2}{*}{2002} & & & \\
\hline & & & $2005 / 06$ \\
\hline \multirow[t]{4}{*}{2006} & & & \\
\hline & & & $2007 / 08$ \\
\hline & 2009/10 & 2008 & $2008 / 09$ \\
\hline & & 2010 & $2010 / 11$ \\
\hline \multirow[t]{2}{*}{2013} & & 2011 & $2011 / 12$ \\
\hline & $2014 / 15$ & 2013 & \\
\hline 2015 & $2015 / 16$ & & \\
\hline
\end{tabular}


el promedio mensual de los puntos de grilla que definen el sector norte del Mar de Bellingshausen, correspondiente a un área delimitada desde los $65^{\circ} \mathrm{S}$ al hasta el límite continental y desde los $78^{\circ} \mathrm{O}$ hasta la costa oeste de la PA. Se calcularon las anomalías de los promedios mensuales respecto al promedio dado por el periodo 1970-2000. Luego, a los resultados mensuales se les aplicó un filtrado exponencial (ver anexo) que elimina la variabilidad intermensual, pero mantiene el comportamiento estacional y anual.

\section{ANÁLISIS ATMOSFÉRICO ALREDEDOR DE LA PENÍNSULA ANTÁRTICA}

En la Fig. 1 se muestran los resultados de los compuestos para abril-agosto, en el lado izquierdo se presentan los análisis correspondientes a los episodios El Niño y al lado derecho para La Niña. Puede verse que durante El Niño (La Niña) se establece un centro de anomalías positivas (negativa) en el campo de superficie (presión atmosférica) y de altura geopotencial de 500 $h \mathrm{~Pa}$, al oeste de la PA. Estas anomalías positivas (negativas) revelan un predominio de dorsales o altas de bloqueo (bajas y vaguadas), es decir, una mayor actividad anticiclónica (ciclónica) a lo normal al oeste de la península; o bien, una disminución de la actividad ciclónica (anticiclónica), lo que puede traducirse en menor precipitación. Los análisis de los mapas compuestos de espesores 1000$500 \mathrm{hPa}$ (no mostrados) indican que durante los episodios El Niño predomina una masa de aire cálido en la región en donde se ubican las anomalías positivas de los campos de superficie y $500 \mathrm{hPa}$, y una masa de aire frio sobre y al este de la península sobre la región suroccidental del Océano Atlántico.

También, durante los eventos El Niño tiene lugar un sector de anomalías negativas en la región oriental del Océano Pacífico al oeste de la costa central de Chile, en los campos de presión en superficie, $500 \mathrm{hPa}$ y espesor 1000-500 hPa. Esto es reflejo del debilitamiento de la Alta presión permanente del Pacífico Sur y de la mayor actividad ciclónica y frontal que puede ocasionar años lluviosos en la región centro-sur Chile (Montecinos \& Aceituno 2003). Para el caso de eventos de La Niña, el análisis compuesto del campo de espesores revela un predominio de una masa relativamente cálida afectando la península, centrado sobre el Mar de Amundsen. La casi superposición de las áreas de anomalías en superficie y altura indica la característica barotrópica-equivalente de la atmósfera. Por otra parte, las anomalías positivas (cálido) del espesor durante El Niño en el sector sur austral del Océano Pacífico revela la característica de bloqueo de las regiones de las altas presiones (sector de anomalías positivas). La presencia de las anomalías positivas centrada aproximadamente en los $110^{\circ} \mathrm{O}$ y 57 ㅇs, indican que la PA queda bajo el sector delantero de la dorsal (o del centro de alta presión), por lo que prevalece un flujo del suroeste en esta región, es decir, masa de aire frío desplazándose hacia el noroeste. El mapa de espesores refleja este hecho al revelar una masa ligeramente más fría afectando la Península durante episodios de El Niño. Los resultados de los compuestos para primavera-verano son similares a los descritos para otoño-invierno, salvo que las anomalías positivas y negativas están más desplazadas al oeste en el caso de El Niño. Esto sugiere que en sector del Mar de Bellingshausen/Amundsen (y Weddell) hay menor actividad anticiclónica y/o mayor ciclónica. En el caso de La Niña, los compuestos de superficie y $500 \mathrm{hPa}$ muestran un centro de anomalías negativa más definido al oeste del Paso Drake. En este caso, prevalece la actividad ciclónica y/o hay menos actividad anticiclónica en el sector suroeste del Pacífico, incluyendo la PA y la región austral de Sudamérica.

El mapa compuesto de las anomalías de la velocidad del viento al nivel de $250 \mathrm{hPa}$ (Fi.a 1c) muestra para los eventos El Niño la intensificación de la corriente en chorro subtropical y el debilitamiento de la corriente en chorro polar en el Océano Pacífico Sur, situación que se revierte para el caso de los eventos La Niña (Chen et al. 1996, Teleti \& Luis, 2016). El primer caso (El Niño), da cuenta de una mayor actividad ciclónica y frontal en las latitudes medias con proyecciones hacia la zona central y sur de Chile, y menor actividad en la zona austral y de la PA. En el segundo caso (La Niña), la intensificación de la corriente en chorro polar revela una mayor actividad ciclónica en el océano Pacífico Sur con proyección hacia el sector austral del continente Sudamericano que no necesariamente alcanza la 
a)

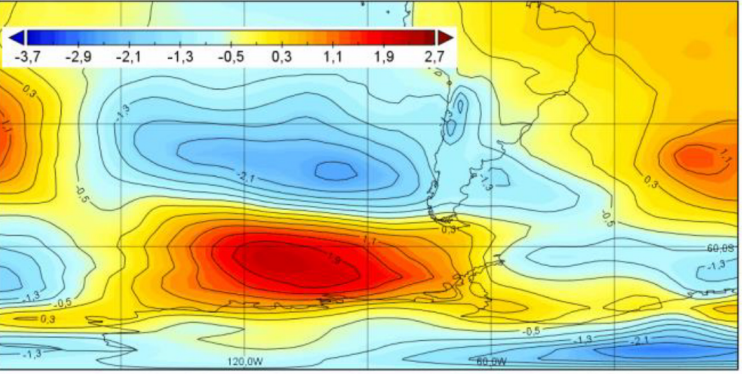

Anomalías de la presión atmosférica a nivel medio del mar ( $\mathrm{hPa}$ )

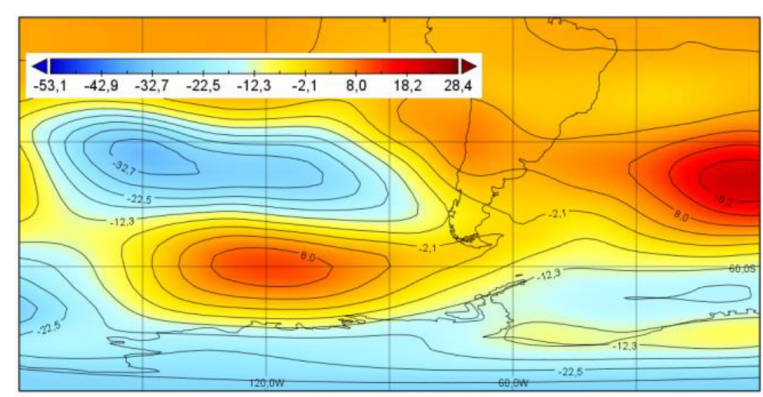

b)

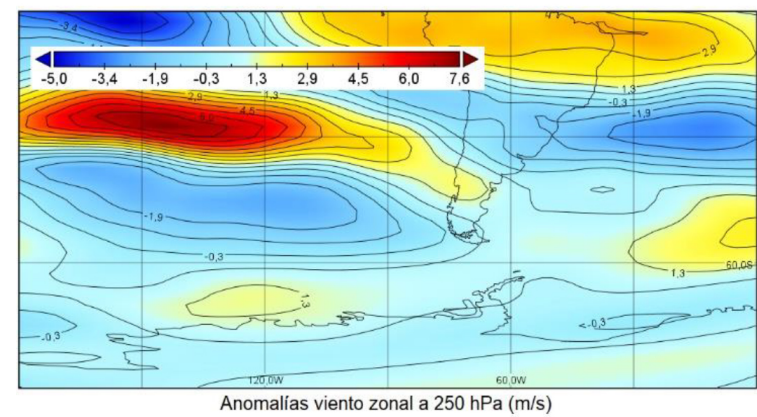

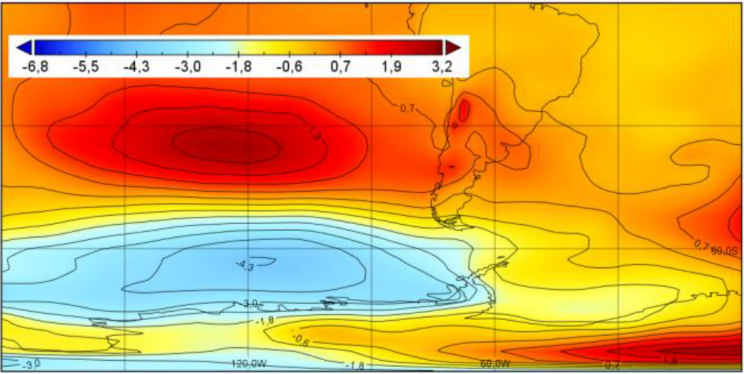

Anomalias de la presión atmosférica a nivel medio delmar (hPa)

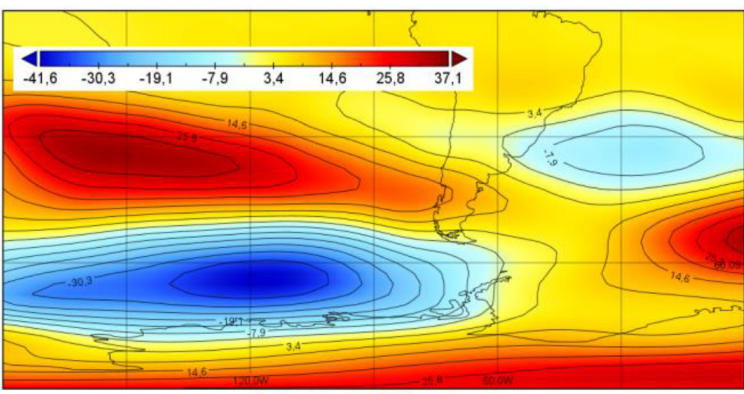

Anomalias de $500 \mathrm{hPa}(\mathrm{gpm})$

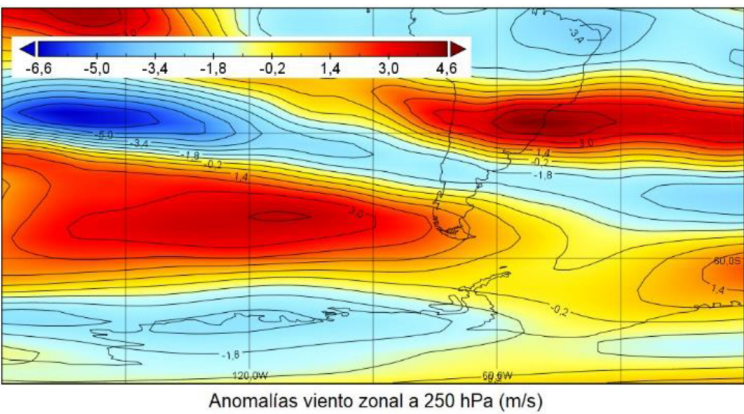

Fig. 1. Anomalías compuestas del periodo abril-agosto para los episodios El Niño (izquierda; 1982,1987 y 1997,2015$)$ y La Niña (derecha; 1988, 1996, 1998, 2008, 2010, 2011, 2013). a) Anomalías de la presión atmosférica a nivel medio del mar, b) Anomalías de la altura geopotencial de $500 \mathrm{hPa}$, y c) Anomalías del viento zonal (en ms $\mathrm{m}^{-1}$ ) a $250 \mathrm{hPa}$. Obtenidos de la base de datos de reanálisis del NCEP (www.esrl.noaa.gov/psd/cgi-bin/data/composites/plot20thc.v2.pl).

PA. Por otra parte, Teleti y Luis (2016) sugieren que, con un chorro subtropical intensificado junto con el chorro polar debilitado, más calor es transportado hacia el polo en el sector del Océano Pacífico Austral resultando en menos hielo marino, mientras que un impacto contrario ocurriría en el sector del Atlántico sur.

Los compuestos de anomalías para primavera-verano del viento zonal a $250 \mathrm{hPa}$ para $\mathrm{El}$ Niño, sigue mostrando una corriente en chorro subtropital (polar) intensificada (debilitada), pero desplazada más al sur que en otoño-invierno. Esto indicaría un desplazamiento hacia el sur de la actividad ciclónica y frontal.
En cambio, para el caso de La Niña primaveraverano la corriente en chorro subtropical se ve intensifica y la polar debilitada en el sector del Pacífico, lo que sugiere menor actividad ciclónica en el sector austral y de la PA.

\section{COMPORTAMIENTO METEOROLÓGICO EN LAS BASES CHILENAS}

La Fig. 2 muestra la variabilidad de las variables atmosféricas de la presión, la temperatura mínima y de la precipitación; en términos de sus anomalías estandarizadas junto al indice IOS. Se calcularon las anomalías respecto al 
promedio dado por el periodo 1970-2000. Todas las curvas fueron filtradas aplicando un filtrado exponencial (ver anexo) que elimina la variabilidad intermensual, pero mantiene el comportamiento estacional y anual. Los resultados muestran que durante los eventos de El Niño la presión presenta un aumento (anomalías positivas), mientras que la precipitación registra una disminución, coincidiendo en algunos casos los máximos de presión y mínimos de precipitación con el instante de mayor intensidad del IOS. Así, las anomalías de la presión y precipitación presentan un comportamiento opuesto (Fig. 2). Sin embargo, y como era de esperarse de acuerdo al resultado de las anomalías compuestas de los mapas de reanálisis (Fig. 1), la relación estadística (correlación) entre las variables meteorológicas y de éstas con el IOS es casi nula. Esto, en parte, puede deberse al comportamiento de estas variables meteorológicas durante el episodio El Niño 91/95 y de La Niña 88/89 y 98/00 (Fig. 3). En particular, durante La Niña Jun88-Jul89 la precipitación experimenta un aumento mientras que la presión local disminuye; en cambio, para La Niña Oct98-Ene01 y Nov95/Dic96 tiene lugar una disminución de la precipitación y aumento de la presión local. Por otra parte, la temperatura mínima superficial presenta un comportamiento más bien aleatorio respecto a la presión y la precipitación, como también con el IOS. La Fig. 3 muestra el comportamiento de la precipitación en las estaciones chilenas para los eventos de $\mathrm{El}$ Niño y La Niña. Puede verse que en general la precipitación disminuye con El Niño, mostrando cierta correlación, pero no así para el caso de los eventos La Niña. Por esto que no se encuentra una correlación estadística entre IOS y la precipitación en el sector norte de la PA.

\section{COMPORTAMIENTO DEL HIELO MARINO AL OESTE DE LA PENÍNSULA ANTÁRTICA}

Como se indicó, uno de los impactos de ENOS es sobre la extensión y concentración del hielo marino (Carleton, 1988; Gloersen, 1995; Simmonds \& Jacka, 1995). La Fig. 4 muestra el comportamiento anual de la anomalía porcentual de la $\mathrm{CHM}$ en el sector norte del Mar de Bellingshausen. En general, la CHM aumenta (disminuye) durante los eventos El Niño (La Niña), aunque los datos no muestran una correlación estadísticamente significativa. Este comportamiento está relacionado con la disminución de la actividad ciclónica en el sector sureste del Pacifico austral, lo cual, en términos de anomalías, aparece un área de anomalías de alta presión (Fig. 1). Esto, en promedio, significa que durante los años El Niño (La Niña) hay mayor frecuencia de vientos del suroeste (noroeste) afectando el sector oeste de la PA como lo muestra el análisis compuesto de las anomalías del campo de presión a nivel medio del mar (Fig. 1a), del cual se puede deducir la circulación anticiclónica con vientos del suroeste en el margen este de las anomalías positivas, al oeste de la PA. En la Fig. 4 , se destaca además (encerrados en círculos) los meses de octubre, noviembre y diciembre de 2016. Durante la primavera de 2016 se registró un mínimo histórico de la extensión del hielo marino en la Antártica (Stuecker et al. 2017; Turner et al. 2017). Si bien la CHM no presenta anomalías de valores históricos en el sector del Mar de Belingshausen, sí coinciden con el hecho de un mínimo en CHM en la primavera de 2016, y con el cambio de la extensión del hielo marino en el sector del Mar de Amundsen y Bellingshausen del análisis realizado por Turner et al. (2017). En la Fig. 4 también se señala una anomalía negativa de $\mathrm{CHM}$ de $47 \%$ que ocurre en junio de 2017 y aparece como la más alta del registro analizado.

La Fig. 5 muestra las anomalías de la CHM para primavera (septiembre-octubre-noviembre) para el evento El Niño-La Niña 1997/98 construido de la página Climate Reanalyzer (http:// cci-reanalyzer.org), Climate Change Institute, University of Maine, USA. Los análisis fueron hechos a partir del ensamblaje de los modelos de reanálisis de tercera generación de ERA-interim, CFSR, MERRA y JRA-55 (European Reanalysis, Climate Forecasting System Reanalysis, ModernEra Reanalysis Retropestive Analysis and Japanese 55-year Reanalysis). Análisis individuales de cada modelo resuelven similares resultados. Para ilustrar el comportamiento de la CHM, se eligió el evento $1997 / 98$ por ser uno de los eventos calificados como fuerte y mostrar una mejor señal en la presión atmosférica medida en la estación Frei (Fig. 2a). La primavera de 1997 muestra mayor CHM que en el 

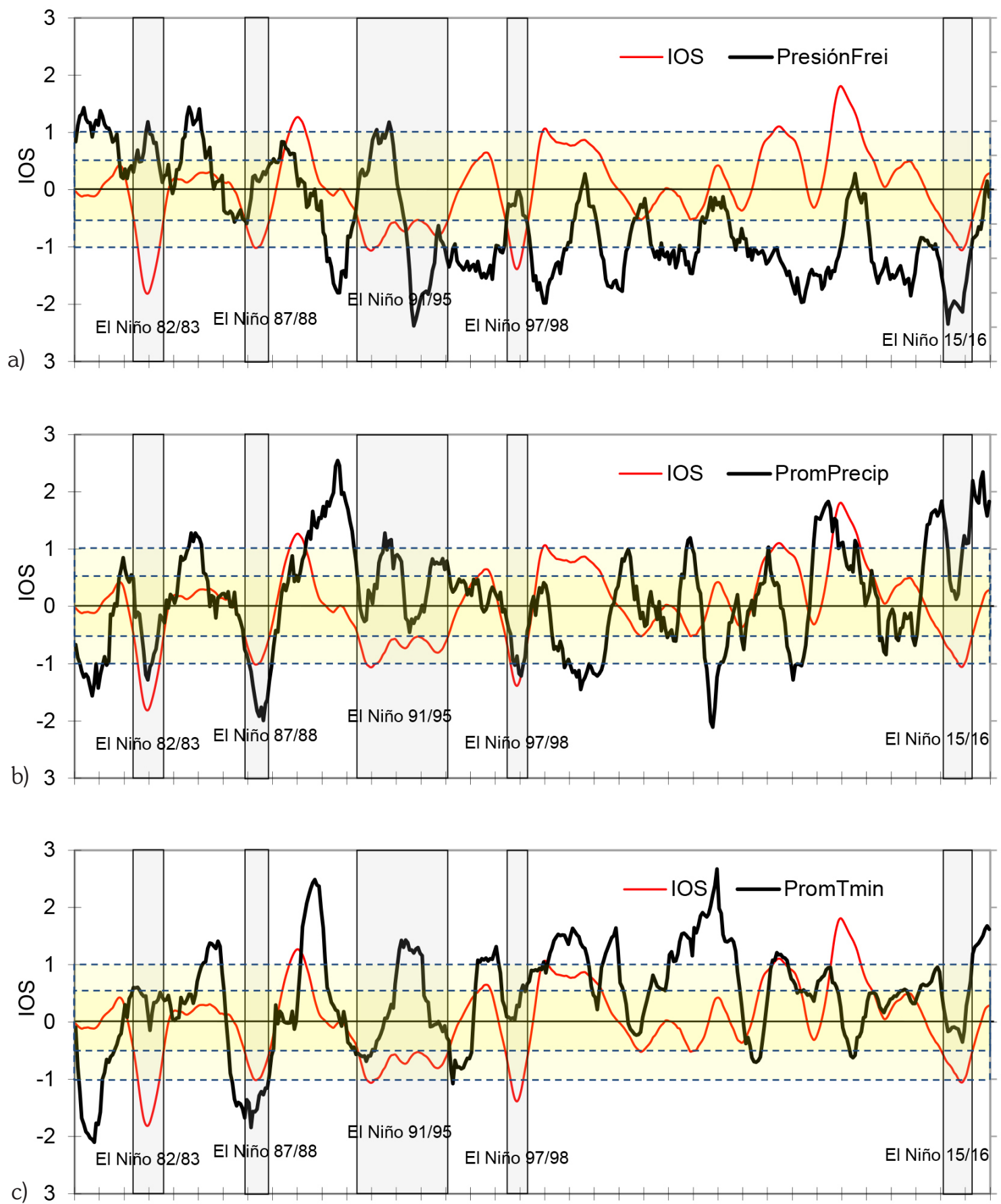

Fig. 2. Comportamiento del Índice Oscilación del Sur (IOS) junto al comportamiento de las anomalías de presión atmosférica en la estación Frei (a), de la precipitación (b) y la temperatura mínima del aire (a) de las estaciones Eduardo Frei, Prat y O'Higgins. Líneas segmentadas representan los valores -1.0. -0.5, +0.5, +1.0 del IOS. 

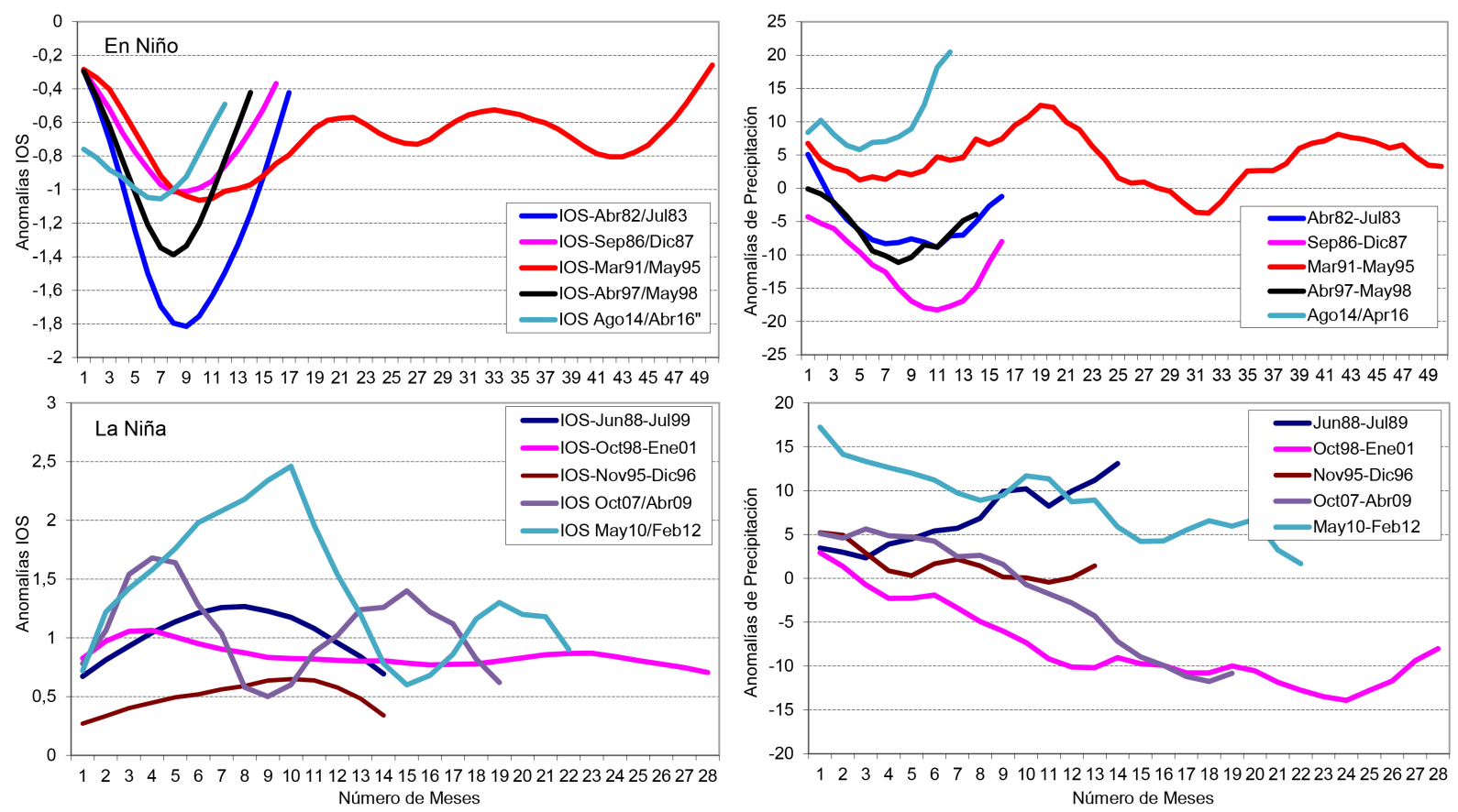

Fig. 3. Arriba (a) Eventos El Niño a partir del mes 1 (inicio, IOS > 0,5) junto al comportamiento de la precipitación media acumulada (anomalías) en las bases antárticas Frei, Prat y O'Higgins. Abajo (b) lo mismo, pero para eventos La Niña.

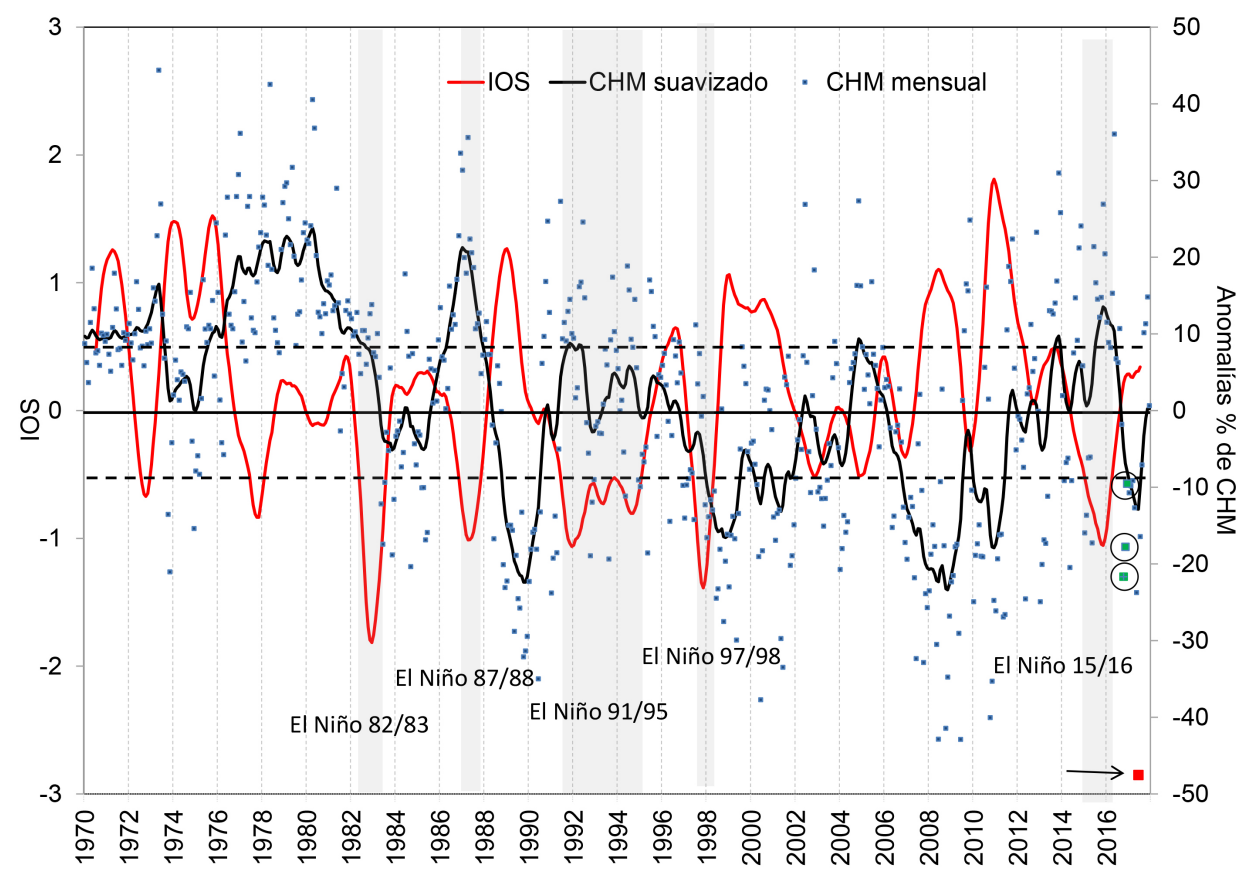

Fig. 4. Comportamiento del Índice de Oscilación del Sur (IOS, eje izquierdo) junto a las anomalías \% de la concentración de hielo marino (CHM) en el sector norte del Mar de Bellingshausen. Ambas curvas son el resultado del filtrado exponencial (ver Anexo). Las líneas segmentadas representan los valores -0.5 y +0.5 del IOS. Valores mensuales de CHM destacados en círculos, corresponden a los meses de octubre, noviembre y diciembre de 2016.

El punto rojo destacado con una flecha corresponde a la anomalía de la CHM de junio 2017. 


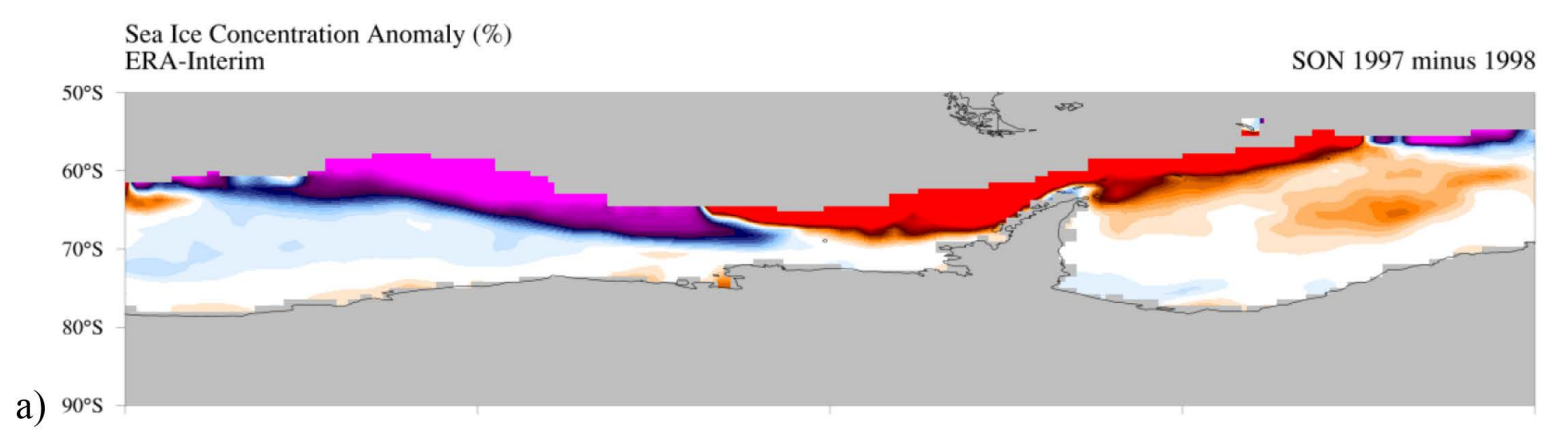

ERA-Interim

SON 1997 minus 1979-2015

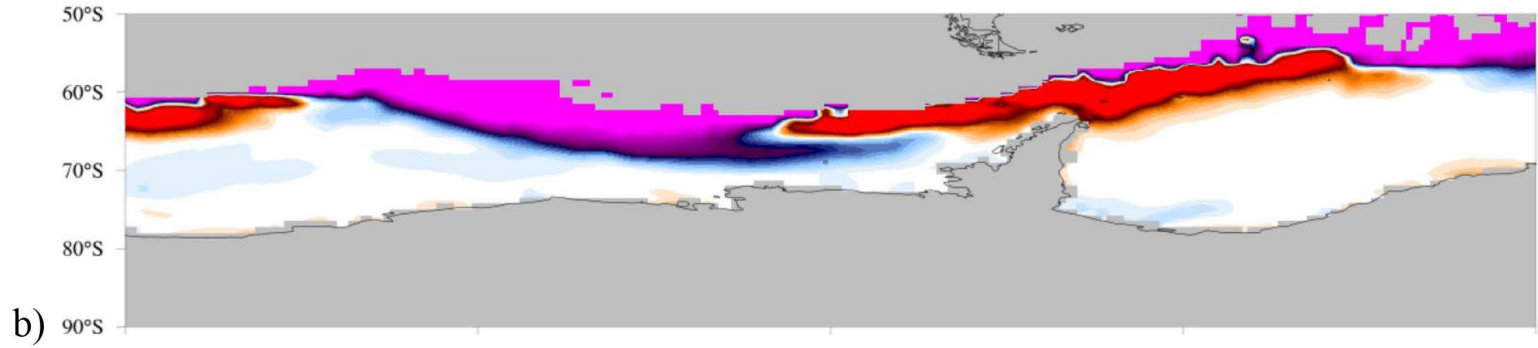

ERA-Interim

SON 1998 minus 1979-2015

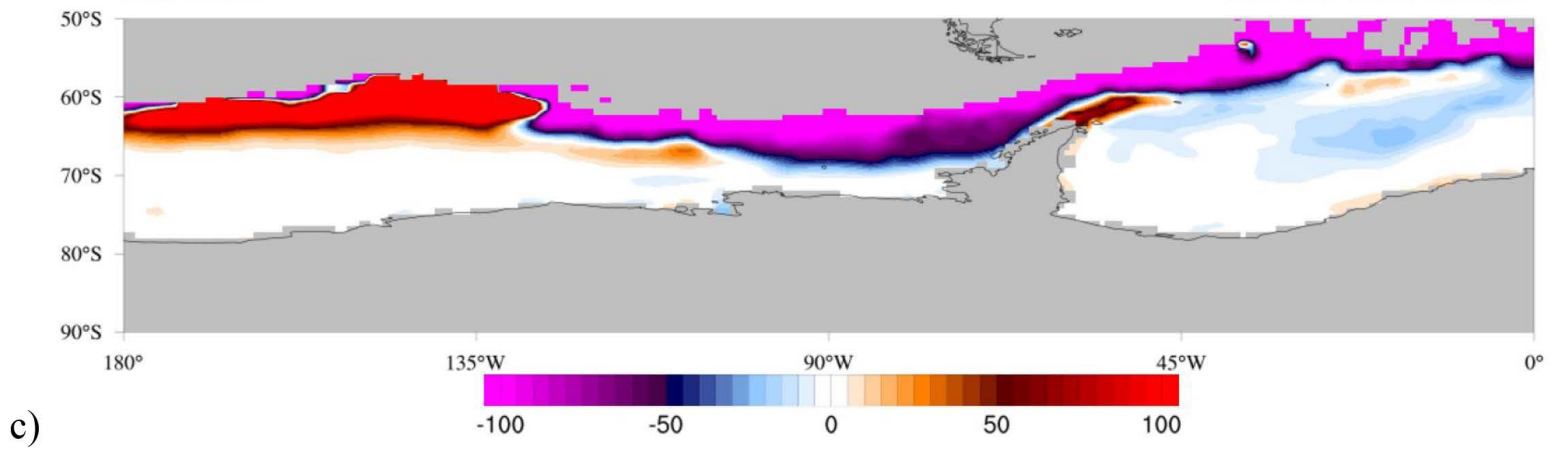

Fig. 5. Diferencias de las anomalías de la concentración de hielo marino en primavera (OND) entre el evento El Niño 1997 y La Niña 1998 (a) junto a la diferencia con respecto a la media dada por el periodo 1979-2000 para El Niño (b) y La Niña (c).

1998 y que el promedio 1979-2000 en el sector oeste de la PA, incluyendo el sector norte del Mar de Bellingshausen. Por el contrario, la primavera de 1998 (con año de La Niña) muestra menor CHM que el promedio 1979-2000 en el sector norte del Mar de Bellingshausen y Amundsen e incluso del Mar de Weddell. Esto es concordante con el análisis de anomalías compuestas que revela que los vientos del suroeste (noroeste) son más frecuentes en el sector sureste del Océano Pacífico Austral, al oeste de la PA, durante El Niño (La Niña).

Note que la Fig. 5 además revela que mientras la $\mathrm{CHM}$ disminuye en el Mar de Bellingshausen, aumenta en el sector norte y noroeste del Mar de Ross y Mar de Amundsen. Este comportamiento está relacionado con la intensidad y posición del centro de Baja presión del Mar de Amundsen/ Bellingshausen (Amundsen/Bellingshausen Sea Low, ABSL), la que para años La Niña está localizada más al oeste, hacia las cercanías del Mar de Ross y, por el contrario, para años El Niño está desplazada más cerca del Mar de Bellingshausen (Turner et al. 2013). La Fig. 6 muestra la correlación lineal entre El Niño 3.4 y las anomalías de la presión a nivel medio del mar para el periodo 1980-2000 construido a partir de la reanálisis del NCEP (www. 
esrl.noaa.gov/psd/data/correlation). Se observa una fuerte correlación positiva, i.e., eventos El Niño está asociado a anomalías positiva de la presión en el sector suroriental del Océano Pacífico, esto es en el sector donde ocurre la variabilidad de ABSL. Las correlaciones son mayores durante la primaveraverano (Fig. 6c) que durante el periodo otoñoinvierno (Fig. 6b). La Fig. 7 muestra un esquema conceptual en donde se describe la relación entre ENOS y ABSL, descrita anteriormente.

La variabilidad de la posición de la ABSL influye en la climatología superficial controlando el viento meridional en el sector de la Antártica Occidental (Hosking et al. 2013, 2015). Una ABSL más intensa puede asociarse con La Niña lo que puede influenciar un predominio de flujo con componente del norte en el sector del Mar de Bellingshausen y del sur el sector del Mar de Ross. Por el contrario, una ABSL asociado a El Niño puede favorecer flujo con componente del sur en el sector del Mar de Bellingshausen y del norte en el sector del Mar de Ross (Karoly, 1989; LachlanCope \& Connolley, 2006; Song et al. 2011). Sinópticamente, durante El Niño menor frecuencia de actividad ciclónica en el sector sureste del Pacífico se traduce en menor frecuencia de vientos del noroeste afectando el Mar de Bellingshausen, favoreciendo la propagación del hielo marino hacia el norte. Por el contrario, durante La Niña puede ocurrir mayor actividad ciclónica al oeste de la PA con mayor prevalencia de vientos del noroeste frenando la propagación hacia el norte de la formación de hielo marino.

\section{CONCLUSIONES}

La PA se encuentra a más de $6000 \mathrm{~km}$ del área del Ecuador donde tiene lugar las variaciones de la temperatura de la superficie del mar conocido como El Niño y La Niña. Las alteraciones

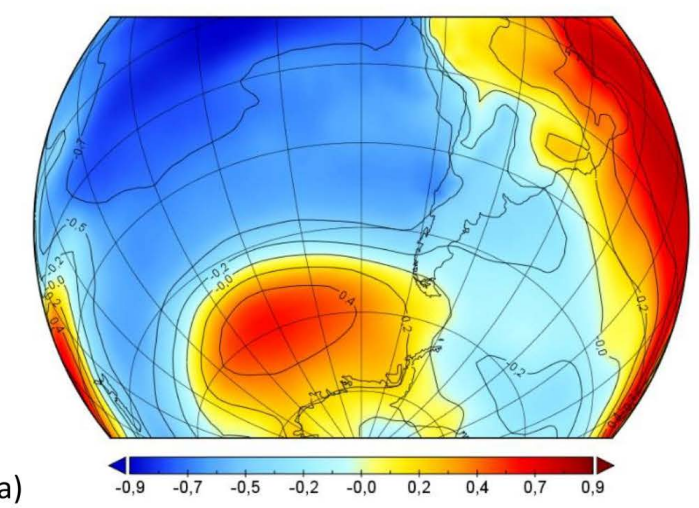

b)
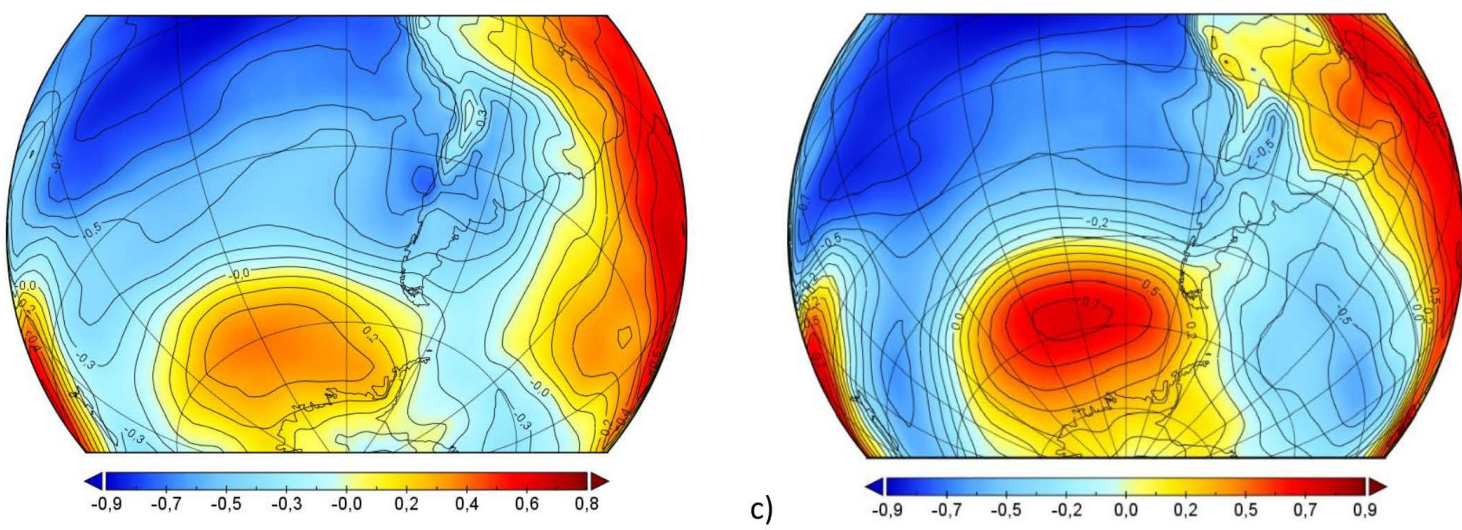

Fig. 6. Correlación lineal entre El Niño 3.4 y la presión a nivel medio del mar para el periodo 1980-2000. a) Anual, b) otoño-invierno (abril-agosto) y c) verano-otoño (septiembre-marzo). Mapas construidos de los reanálisis del NCEP (página web https://www.esrl.noaa.gov/psd/data/correlation/). 

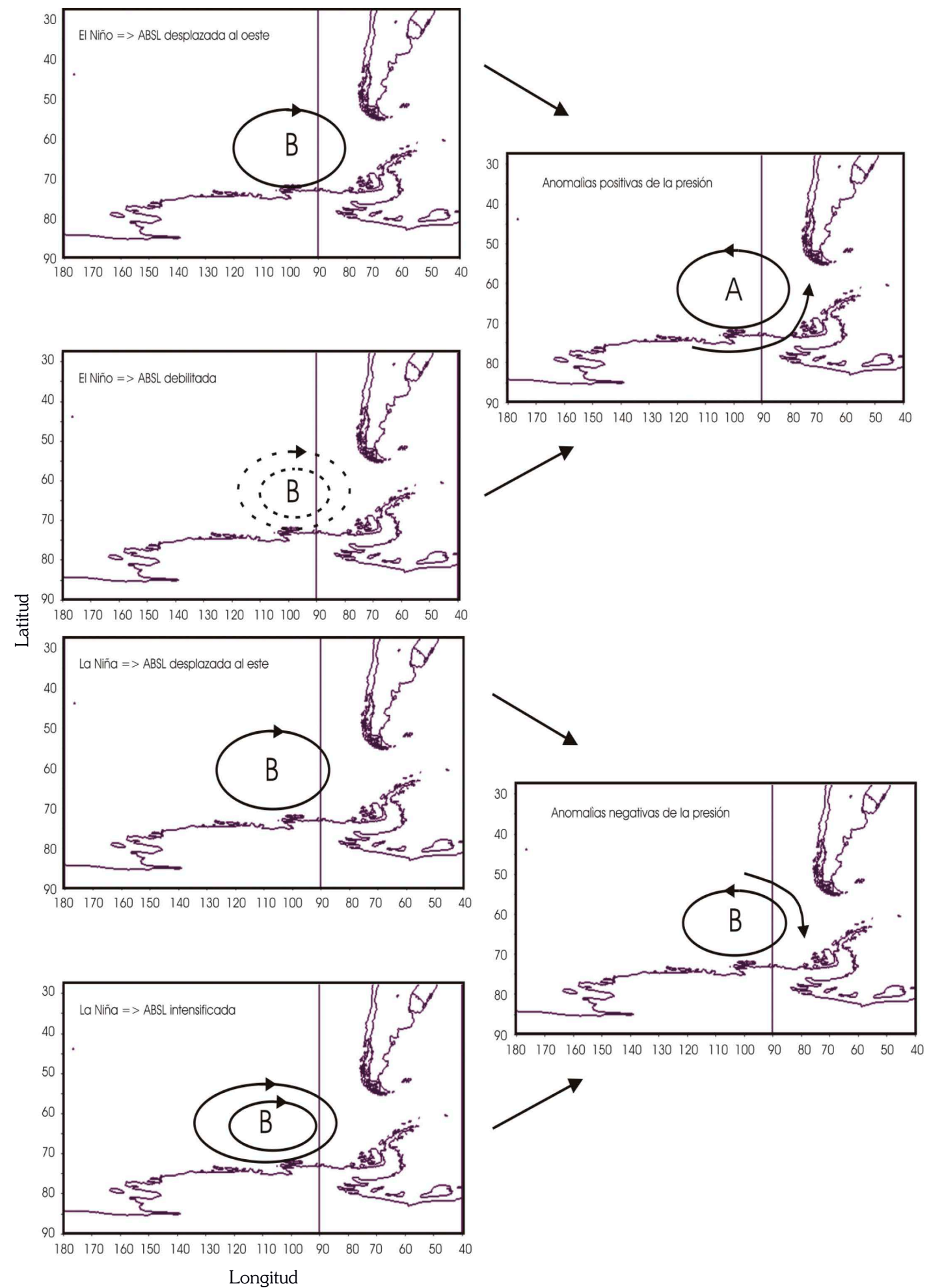

Fig. 7. Esquema conceptual de la relación entre el centro de baja presión de Amundsen/ Bellingshausen (ABSL) y El Niño Oscilación del Sur (ENOS). 
atmosféricas asociadas alcanzan marginalmente a la PA, de allí que la señal de ENOS, en términos de los impactos en el comportamiento de las variables meteorológicas en esta área, no se manifiesten del todo. Sinópticamente, durante un episodio de $\mathrm{El}$ Niño se observa que predomina una anomalía de alta presión en el sector sureste del Océano Pacifico, al oeste de la PA (Figs. 1 y 7). Las anomalías positivas de la presión superficial que experimentan las estaciones chilenas antárticas, tal como lo muestra la estación Freí, es una manifestación del centro de alta presión que se desarrolla al oeste de la península.

Climatológicamente, al oeste de la Península se ubica un centro de bajas presiones que forma parte de la vaguada circumpolar que rodea el continente. En particular, al oeste de la PA encontramos el centro de baja presión de Amundsen/Bellingshausen (ABSL). Estos centros de baja presión son regiones que aparecen climatológicamente como resultado de una mayor actividad ciclónica asociada principalmente a centros depresionarios y perturbaciones frontales que se desplazan hacia el sureste, y en parte a la actividad de ciclones a mesoescala que tiene lugar alrededor de la Antártica, en particular en el sector Pacífico (Carrasco et al. 2003; Pezza et al. 2015). Las anomalías positivas, tanto en superficie como en altura, reflejan una menor actividad ciclónica (y frontal) que la media, o bien, el predominio de una circulación anticiclónica (que se manifiesta como un centro de alta presión o una dorsal). Esto sugiere una relación entre la variabilidad de la ABSL y ENOS (Fig. 7). La disminución de la precipitación en la PA está asociada a la menor actividad ciclónica y frontal que se traduce en un predominio de una circulación anticiclónica en el sector sureste del Pacifico Sur, al oeste de la PA (Figs. 1 y 7). Las anomalías negativas de los análisis compuestos de El Niño indican que la actividad ciclónica estuvo mayormente ubicada en las latitudes medias y al norte del sector del Mar de Ross.

La corriente en chorro polar en promedio se mantiene al sur de Australia - Nueva Zelanda manifestando que durante el episodio El Niño la actividad frontal afectó mayormente al norte del sector de Mar de Ross. Chen et al. (1996) concluyeron que en condiciones de El Niño la corriente en chorro subtropical es más intensa y desplazada hacia el este, mientras que la corriente en chorro polar se debilita y se ubica más alejado (al oeste) de la región austral de Sudamérica y de la PA (ver también Teleti \& Luis, 2016). La menor precipitación registrada por las estaciones chilenas antárticas es el reflejo del desplazamiento de la actividad frontal hacia el sector del Mar de Ross alejándose de la Península. Las señales de Las señales de El Niño, en términos de sus efectos en la precipitación, temperatura y presión atmosférica en las estaciones antártica chilenas localizadas en el sector norte de la PA; son más claramente observadas que las señales de La Niña.

Un impacto de ENOS es sobre la formación del hielo marino en la Antártida. En particular en el sector oeste de la PA, en el Mar de Bellingshausen y Amundsen, la formación y extensión hacia el norte del hielo marino puede verse frenada cuando prevalecen vientos del noroeste en el sector, situación que se da con eventos La Niña. Por el contrario, la formación y desplazamiento del hielo marino puede verse favorecido cuando la frecuencia de vientos del noroeste es baja y débil o incluso la circulación del viento sea del oeste y suroeste, situación que ocurre en los eventos El Niño.

\section{AGRADECIMIENTOS}

Este trabajo fue parcialmente financiado por el proyecto Anillo ARTG02-2006 y por el proyecto Fondecyt $\mathrm{N}^{\circ} 1151034$. Se agradece a dos revisores anónimos que con sus comentarios ayudaron a mejorar el original de este artículo.

\section{LITERATURA CITADA}

Bromwich, D. H., Rogers, A. N., Kallberg, P., Cullather, R. I., Whiteand, J. W. C., \& Kreutz. K.J. (2000). ECWRF analysis and reanalysis depiction of ENSO signal in Antarctic precipitation. Journal of Climate, 13, 1406-1420.

Carleton, A. M. (1988). Sea ice - atmosphere signal of the Southern Oscillation in the Weddell Sea, Antarctica. Journal of Climate, 1, 379-388

Chen, B., Smith, S. R., \& Bromwich, D.H. (1996). Evolution of the Tropospheric Split Jet over the South Pacific Ocean during the 19861989 ENSO cycle. Monthly Weather 
Review, 124, 1711-1731.

Chen, W. Y. (1982). Assessment of Southern Oscillation sea level pressure indices. Mon. Weather Review, 110, 800-807.

Carrasco, J. F., Bromwich, D. H., \& Monoghan, A. J. (2003). Distribution and characteristics of mesoscale cyclones in the Antarctic: Ross Sea eastward to the Weddell Sea. Monthly Weather Review, 131, 289-301.

Cullather, R. I., Bromwich, D. H., \& Woert, M. L. (1996). Interannual variation in Antarctic precipitation related to El Niño-Southern Oscillation. Journal of Geophysical Research, 101(D14), 19.109-19.118.

Gloersen, P. (1995). Modulation of hemispheric sea-ice cover by ENSO events, Nature, 373, 503-506.

Karoly, D. J. (1989), Southern Hemisphere circulation features associated with El NiñoSouthern Oscillation events, Journal of Climatology, 2, 1239-1252.

Lachlan-Cope, T. A., \& Connolley, W. M. (2006), Teleconnections between the tropical Pacific and the AmundsenBellinghausens Sea: Role of the $\mathrm{El}$ Niño/Southern Oscillation, Journal of Geophysical Research, 111, D23101, doi:10.1029/2005JD006386.

Mo, K. C., Pfaendtner J., \& Kalnay, E. (1987). A GCM study on the maintenance of the June 1982 blocking in the Southern Hemisphere. Journal of Atmospheric Sciences, 44, 1123-1142.

Montecinos, A., \& Aceituno, P. (2003). Seasonality of the ENSO-Related rainfall variability in central Chile and associated circulation anomalies. Journal of Climate, 16, 281296.

Pezza, A, Sadler, K., Uotila, P., Vihma, T., Mesquita M. D. S., \& Reid P. (2015). Southern Hemisphere strong polar mesoscale cyclones in high-resolution datasets. Climate Dynamics. Doi 10.1007/s00382015-2925-2.

Renwick, J. A. (1998) ENSO-related variability in the frequency of South Pacific blocking. Monthly Weather Review, 126, 31173123.

Rosenblüth, B., Fuenzalida, H. A., \& Aceituno,
P. (1997). Recent temperatures variations in southern South America. International Journal of Climatology, 17, 67-85.

Savage, M., Weidner, G., \& Stearns, C. (1988). A diagnostic study of the influence of a gravity wave upon regional weather, Monthly Weather Review, 116, 347-357, 1988.

Simmonds, I., \& Jacka, T.H. (1995). Relationships between interannual variability of Antarctic sea ice and Southern Oscillation. Journal of Climatology, 8, 637-647.

Smith, S. R., \& Stearns, C. R. (1993a). Antarctic pressure and temperature anomalies surrounding the minimum in the Southern Oscillation index. Journal of Geophysical Research, 98, 13.071-13.083.

Smith, S. R., \& Stearns, C. R. (1993b). Antarctic Climate Anomalies Surrounding the Minimum in the Southern Oscillation index. In D. H. Bromwich \& C. R. Stearns (Eds.), Antarctic Research Series: Antarctic Meteorology and Climatology, 61, 149174.

Song, H.-J., Choi, E., Lim, G.-H., Kim, Y. H., Kug, J.-S., \& Yeh S.-W. (2011), The central Pacific as the export region of the El Niño-Southern Oscillation sea surface temperature anomaly to Antarctic sea ice, Journal of Geophysical Research, 116, D21113, doi:10.1029/2011JD015645.

Stuecker, M. F., Bitz, C. M., \& Armour, K. C. (2017). Conditions leading to unprecedented low Antarctic sea ice extent during the 2016 austral season. Geophysical Research Letters, 44, 9008-9019, doi:10.1002/2017GL074691.

Teleti, P. R. \& Luis, A. J. (2016). The role of the Southern Hemisphere Polar Cell on Antarctic Sea Ice Variability. International Journal of Geosciences, 7, 120-134.

Office Hadley Centre sea ice and sea surface temperature data set, version 2: 1 . Sea ice concentrations. Journal of Geophysical Research - Atmosphere, 119, 2864-2889.

Trenberth, K. E. (1997). The Definition of El Niño. Bull. Am. Meteorol. Soc. 78, 2771-2777.

Turner, J. (2004). Review: The El Niño-Southern Oscillation and Antarctica. International Journal of Climatology, 24, 1-31. 
Turner, J., Phillips, T., Hosking, J. S., Marshall, G. J., \& Orr, A. (2013). The Amundsen Sea low, International Journal of Climatology, 33(7), 1818-1829.

Turner, J., Phillips, T., Marshall, G. J., Hosking, J. S., Pope, J. O., Bracegirdle, T. J. \& Deb, P. (2017). Unprecedented springtime retreat of Antarctic sea ice in 2016. Geophysical Research Letters, 44, 6868-6875.

Van Loon, H., \& Shea, D.J. (1987). The Southern Oscillation. Part VI: Anomalies of sea level pressure on the Southern Hemisphere and of Pacific sea surface temperature during the development of a warm event. Monthly Weather Review, 115, 370-379.

White, W. B., \& Petersen, R. G. (1996). An Antarctic circumpolar wave in surface pressure, wind, temperature and sea-ice extent, Nature, 380, 699-702.

Yuan, X. (2004) ENSO-related impacts on Antarctic sea ice: a synthesis of phenomenon and mechanisms. Antarctic Science, 16(4), 415-425.

Yuan, X., \& Martionson D. G. (2001). The Antarctic Dipole and its Predictability. Geophysical Research Letters, 28(18), 3609-3612. 


\section{ANEXO}

El filtro exponencial (ver Rosenblüth et al. 1997) toma los datos individuales y los suaviza eliminando la alta variabilidad (diaria, mensual o anual según el análisis) y dejando ver la variabilidad de más largo plazo (mensual, estacional, anual o decadal según el análisis). Utiliza la expresión:

$$
\begin{aligned}
& y_{t}=c \cdot x_{t}+(1-c) \cdot y_{t-1} \quad t=2,3, \ldots \ldots n \\
& z_{t}=c \cdot y_{t}+(1-c) \cdot z_{t-1} \quad t=(n-1),(n-2), \ldots \ldots 1
\end{aligned}
$$

donde (1) y (2) son respectivamente, el primer filtrado de la serie de datos hacia adelante y el segundo filtrado, hacia atrás desde el final de la serie resultante del primer filtrado, permitiendo "suavizar" los datos. El primer valor yt es el promedio de los primeros diez (10) valores de la serie y $z_{t}$ corresponde al valor final del primer suavizado hacía adelante. El grado de suavizado $(c)$ puede variar desde 1 (máximo, reproduce los datos originales) a 0 (da como resultado una línea recta). En este caso, se usó $c=0,11$ lo que permite filtrar las frecuencias altas como la variabilidad intermensual, pero mantiene el comportamiento de largo plazo. El siguiente gráfico ejemplifica el filtrado exponencial.

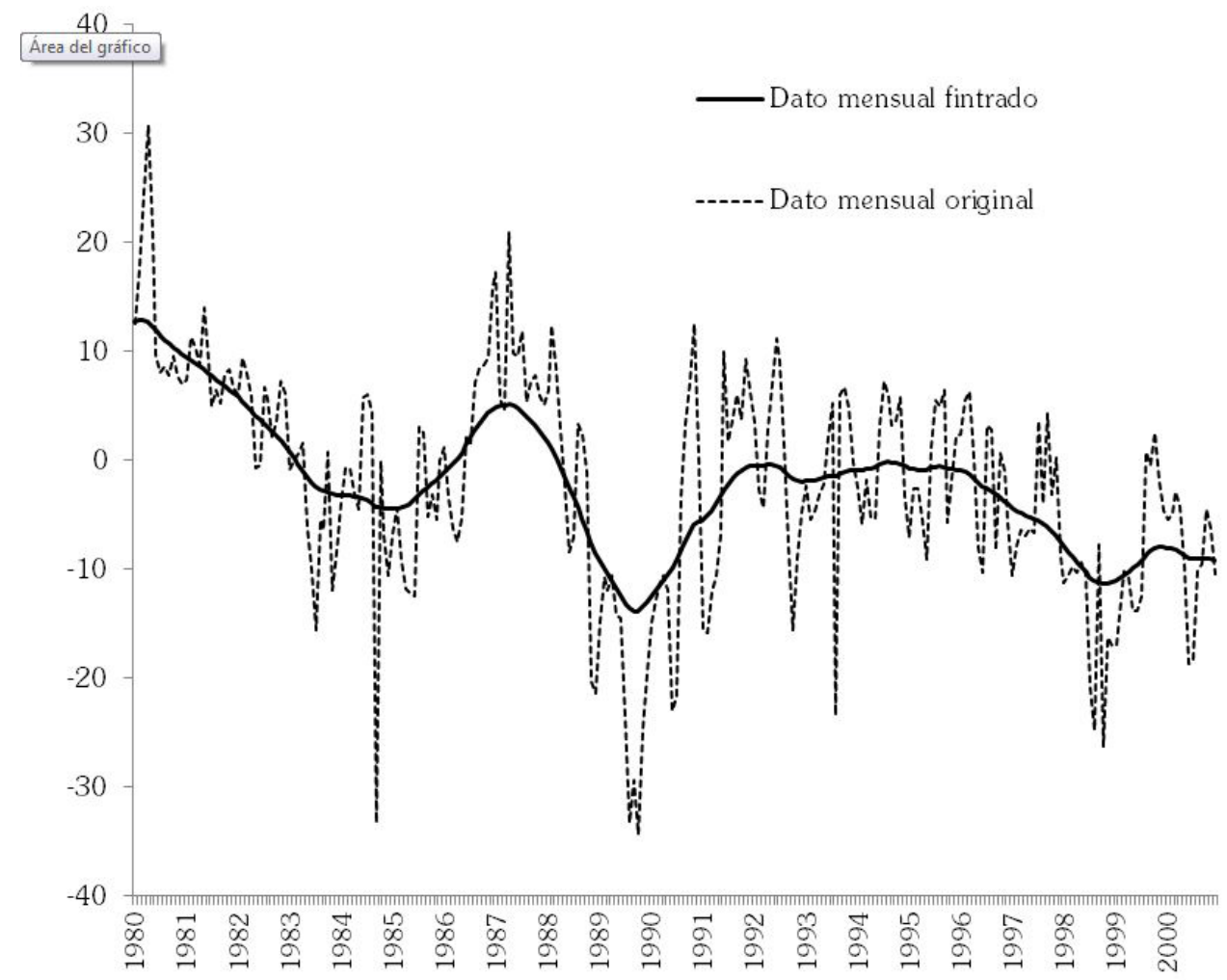


